\title{
The Burden of Illness of Migraine in Canada: New Insights on Humanistic and Economic Cost
}

\author{
Farnaz Amoozegar, Zaeem Khan (D), Mariana Oviedo-Ovando, Stephanie Sauriol, \\ Driss Rochdi
}

\begin{abstract}
Background: The aim of this study was to characterize the burden of illness of migraine in Canada. The primary objective was to estimate the annual direct medical resource use and associated costs in migraine patients who failed at least two prophylactic therapies for migraine. Methods: Adults with at least four migraine days per month and who had failed at least two prophylactic migraine therapies were included. Participation in a clinical trial within 12 months of enrollment was the sole exclusionary criterion. Patient demographic and clinical characteristics, migraine-related treatment and medical history, and direct medical resource utilization were collected through a retrospective medical chart review. Data on patient characteristics, lifestyle factors, treatments, medical resource utilization, out-of-pocket expenses, and indirect costs were collected through a cross-sectional patient survey. The patient survey also included validated patient-reported outcome instruments to assess migraine impact on quality of life and work productivity loss. Results: In total, 287 migraine patients were included. The mean time since migraine diagnosis was 14.3 years and patients experienced a mean of 14.1 migraine days per month. The total estimated annual cost of chronic migraine (CM) was $\$ 25,669$ per patient, while the annual total costs for high-frequency episodic and low-frequency episodic migraine (EM) were estimated to be $\$ 24,885$ and $\$ 15,651$, respectively. Conclusion: Migraine is associated with moderate to severe disability. This results in substantial economic burden, directly from healthcare costs such as prescription medications and indirectly through lost work productivity. We also observed that patients with high-frequency EM experience significant burden, similar to that observed for patients with CM.
\end{abstract}

RÉSUMÉ : Le fardeau de la migraine au Canada : nouvelle perspective des coûts tant humains qu'économiques. Contexte : L'étude visait à caractériser le fardeau de la migraine au Canada, et avait pour objectif principal l'évaluation de l'utilisation annuelle directe des ressources médicales et des coûts associés à l'échec d'au moins deux cures de traitement prophylactique de la migraine. Méthode : Ont été sélectionnés des adultes qui souffraient de migraine au moins quatre jours par mois et qui n'avaient pas été soulagés par au moins deux cures de traitement prophylactique. La participation à un essai clinique au cours des 12 mois précédant l'admission était le seul critère d'exclusion. La collecte de données sur les caractéristiques démographiques et cliniques des patients, les antécédents médicaux, les traitements liés à la migraine, de même que celles sur l'utilisation directe des ressources médicales s'est faite par un examen rétrospectif des dossiers médicaux. Quant aux données sur les caractéristiques des patients, les facteurs liés au mode de vie, les traitements, l'utilisation des ressources médicales, les frais assumés par les patients eux-mêmes et les coûts indirects, elles ont été recueillies à l'aide d'un questionnaire d'enquête transversale remis aux participants. Enfin, l'enquête comprenait également des instruments validés de résultats déclarés par les patients afin que soit évaluées les répercussions de la migraine sur la qualité de vie et la perte de productivité au travail. Résultats : Au total, 287 patients souffrant de migraine ont participé à l'étude. Le temps moyen écoulé depuis la pose du diagnostic de migraine était de 14,3 ans, et les patients éprouvaient en moyenne 14,1 jours de migraine par mois. Le coût annuel total de la migraine chronique a été évalué à $25669 \$$ par patient, et celui de la migraine épisodique fréquente et de la migraine épisodique peu fréquente, à 24885 \$ et à 15651 \$, respectivement. Conclusion : La migraine est associée à une incapacité modérée ou grave, qui se traduit par un important fardeau économique, à la fois direct en raison du coût des soins de santé tels que celui des médicaments sur ordonnance, et indirect en raison de la perte de productivité au travail. Enfin, il ressort de l'étude que les patients souffrant de migraine épisodique fréquente supportent un fardeau important, comparable à celui que subissent les patients souffrant de migraine chronique.

Keywords: Neurology, General, Migraine, Quality of Life, Epidemiology

doi:10.1017/cjn.2021.75

Can J Neurol Sci. 2022; 49: 249-262

\section{INTRODUCTION}

Migraine is a debilitating neurological disorder with a prevalence of around $8.3 \%$ in Canada. It is defined by moderate to severe headache attacks that are often accompanied by neurological symptoms such as sensory and dysautonomic symptoms, including nausea, vomiting, photophobia, and phonophobia. ${ }^{1-4}$ Based on the frequency of headache attacks, migraine can be classified as either episodic migraine (EM), characterized by fewer than 15 headache days per month, or chronic migraine $(\mathrm{CM})$, characterized by 15 or more headache days per month with

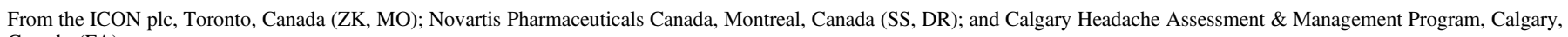
Canada (FA)

Received November 19, 2020. Final Revisions Submitted April 12, 2021. Date of Acceptance April 12, 2021.

Correspondence to: Driss Rochdi, Novartis Pharmaceuticals Canada, Montreal, Canada. Email:driss.rochdi@novartis.com 
at least 8 days being migraine days. ${ }^{1,2,5}$ However, a growing number of studies are suggesting that this classification should be further revised and that EM should be divided in two subgroups: low-frequency EM (1 to 7 monthly migraine days [MMDs])) and high-frequency EM (8 to 14 MMDs), where high-frequency EM has much more in common with $\mathrm{CM}$ in regard to its associated level of disability. ${ }^{6,7}$ During a migraine episode, patients can experience significant disability, moderate to severe pain, limited mobility, and reduced physical and cognitive functioning which considerably impacts the patients' daily life activities, work, and educational opportunities. $^{4,8}$ Migraine is associated with impaired quality of life (QoL), and significant personal, economic, and societal burden. ${ }^{9,10}$ Canadian treatment guidelines include medications for acute migraine attacks and prophylactics for the prevention of migraine. Acute migraine treatment aims to abort a migraine attack or relieve pain and associated symptoms, whereas prophylactic treatments aim at reducing frequency, duration, and severity of attacks.

It is important to understand the burden of illness associated with migraine in Canada in order to address patients' unmet needs. It has been previously reported that the total mean headache-related healthcare costs (including medical resource and medication use), over 3 months, among CM subjects were \$471 Canadian dollars (CAD) $( \pm 1022)$ compared to $\$ 172 \mathrm{CAD}( \pm 920, p<0.001)$ for $\mathrm{EM}$ subjects. ${ }^{11}$ However, the lack of recent data for the economic (direct and indirect) impact and QoL makes it difficult to understand the current burden of migraine among Canadians given that treatment practices have changed over the last decade.

To address the knowledge gaps discussed above, we conducted a cross-sectional observational patient survey and retrospective medical chart review. The overarching aim of this study was to characterize the burden of illness of migraine in Canada, including the clinical, humanistic, and economic burden. The primary objective of this study was to estimate the annual direct medical resource use and associated costs in patients suffering from migraine who have failed at least two prior prophylactic therapies for migraine. The secondary objectives of the study aimed to describe the demographic, clinical, and treatment characteristics, estimate out-of-pocket and indirect costs (lost/missed work; productivity loss) associated with migraine, and to assess the impact of migraine using validated patient-reported outcome (PRO) instruments.

\section{Methods}

This observational study collected data on a cohort of migraine patients attending one of five Canadian migraine centers as outpatient clinic. The target population were adults with a physician-confirmed diagnosis of migraine (at least 12 months prior to study enrollment) and at least four migraine days per month over the 3 months prior to study enrollment. Participants had to have failed at least two prophylactic migraine therapies in the 5 years prior to study enrollment due to tolerability or efficacy reasons. Participants also had to be willing and able to provide informed consent and complete the patient survey. Participation in a clinical trial of an investigational drug at the time of enrollment or within 12 months prior to study enrollment was the sole exclusionary criterion.

Primary and secondary data were collected using a crosssectional patient survey and retrospective medical chart review, respectively. All data were entered electronically and were monitored remotely. Key variables were source data verified during on-site monitoring visits. The data collection period was between January and November 2019. Ethical approval was obtained for all study sites.

\section{Study Variables, Assessment of Outcomes, and Statistical Analyses}

Data on patient demographic and clinical characteristics, migraine-related treatment and medical history, and data on direct medical resource utilization from the previous 12 months since study enrollment were collected from the medical charts. Data on patient characteristics, lifestyle factors, treatments, medical resource utilization (e.g. physiotherapy, massage therapy, and other healthcares resources), out-of-pocket expenses, and indirect costs were collected through the patient survey. The patient survey also included validated PRO instruments to assess the impact of migraine in a standardized approach. Headache Impact Test (HIT-6 ${ }^{\mathrm{TM}}$ ), EQ-5D, and Migraine-Specific Quality of Life Questionnaire (MSQ) were used to assess the impact of migraine on health- and migraine-related QoL. In addition, assessment of disability and work productivity loss due to migraine were performed using the Migraine Disability Assessment (MIDAS) and the Work Productivity and Activity Impairment (WPAI) questionnaires, respectively. Questions from the survey were in reference to the 3 months prior to study enrollment and responses were extrapolated to 12 months.

The statistical analyses were conducted in Statistical Analysis System (SAS) 9.3. All analyses were descriptive in nature. Continuous variables were summarized by means, standard deviations (SDs), medians, and interquartile ranges (IQRs), and categorical variables were summarized by numbers and percentages. All summarized data are presented in aggregate.

Resource use referred to, but was not limited to, all-cause or migraine-specific office visits, emergency room visits, laboratory tests, hospitalizations, neuroimaging, medications used for migraine including opioids, analgesics, antidepressants, and nonpharmacological interventions such as physiotherapy. Costs were calculated for healthcare resource use by the application of unit costs to each resource use estimate. Unit costs were taken from standard and public sources such as the Ontario Schedule of Benefits, Ontario Case Costing Initiative, and Ontario Drug Benefit Formulary. For medications, a weighted average of unit costs across doses available in the formulary provided an average daily cost, which was applied to all patients who took the medication. Monthly costs were calculated by number of migraine days for acute treatments and assumed to be taken for 30 days each month for prophylactic treatments. Annual costs were calculated by yearly duration of use. Cost of medications did not include pharmacy mark-up or dispensing fees. For migraine-specific devices, it was assumed that all devices were reusable and therefore the annual costs were estimated to be unit price of the device irrespective of frequency of use. Total annual costs per patient are presented and all costs were adjusted to 2018 Canadian dollars using the Consumer Price Index as reported by Statistics Canada.

Where available, indirect cost estimates are provided per patient with any utilization and per entire study population. The latter assumes that those who did not report did not experience the indirect cost (i.e. zero dollars). 
Table 1: Demographic and clinical characteristics of migraine patients in Canada $(n=287)$

\begin{tabular}{|c|c|}
\hline Characteristics & $N(\%)$ \\
\hline \multicolumn{2}{|l|}{ Age at study index date ${ }^{1}$} \\
\hline Mean (SD) & $45.5(12.2)$ \\
\hline Median (IQR) & $45.0(36-55)$ \\
\hline Range & $19-78$ \\
\hline \multicolumn{2}{|l|}{$\operatorname{Sex}^{1}$} \\
\hline Male & $42(14.6)$ \\
\hline Female & $245(85.4)$ \\
\hline \multicolumn{2}{|l|}{ Racial/ethnic background $^{2}$} \\
\hline White/Caucasian & $266(92.7)$ \\
\hline Asian & $9(3.1)$ \\
\hline Other & $8(2.8)$ \\
\hline Latin American & $3(1.0)$ \\
\hline Prefer not to answer & $1(0.3)$ \\
\hline \multicolumn{2}{|l|}{ Main daily activity $^{2}$} \\
\hline Employed, full-time & $127(44.3)$ \\
\hline Employed, part-time & $32(11.1)$ \\
\hline Self-employed & $13(4.5)$ \\
\hline Stay at home/homemaker & $13(4.5)$ \\
\hline Student & $7(2.4)$ \\
\hline Retired & $31(10.8)$ \\
\hline Unemployed, due to migraine & $52(18.1)$ \\
\hline $\begin{array}{l}\text { Unemployed, reasons other than } \\
\text { migraine }\end{array}$ & $9(3.1)$ \\
\hline Prefer not to answer & $3(1.0)$ \\
\hline \multicolumn{2}{|l|}{ Marital status $^{2}$} \\
\hline Single (never legally married) & $58(20.2)$ \\
\hline Married (not separated) & $131(45.6)$ \\
\hline Common law & $52(18.1)$ \\
\hline Separated (legally married) & $12(4.2)$ \\
\hline Divorced & $22(7.7)$ \\
\hline Widowed & $6(2.1)$ \\
\hline Other & $4(1.4)$ \\
\hline Prefer not to answer & $2(0.7)$ \\
\hline \multicolumn{2}{|l|}{ Time since migraine diagnosis (years) ${ }^{1}$} \\
\hline Mean (SD) & $14.3(11.8)$ \\
\hline Median (IQR) & $10.2(4.5-22.2)$ \\
\hline \multicolumn{2}{|c|}{ Time since first onset of migraine symptoms ${ }^{1}$} \\
\hline Mean (SD) & $25.8(15.4)$ \\
\hline Median (IQR) & $25.8(13.9-35.9)$ \\
\hline \multicolumn{2}{|l|}{ Type of migraine $^{1}$} \\
\hline Migraine without aura & $62(21.7)$ \\
\hline Migraine with aura & $29(10.1)$ \\
\hline $\begin{array}{l}\text { Chronic migraine without medication } \\
\text { overuse }\end{array}$ & $143(50.0)$ \\
\hline $\begin{array}{l}\text { Chronic migraine with medication } \\
\text { overuse }\end{array}$ & $52(18.2)$ \\
\hline
\end{tabular}

Table 1: (Continued)

\begin{tabular}{l|c}
\hline Characteristics & $\boldsymbol{N}(\%)$ \\
\hline \multicolumn{2}{l|}{ Migraine days per month, over past 3 months $^{1}$} \\
\hline Mean (SD) & $14.1(8.5)$ \\
\hline Median (IQR) & $11(7.0-19.0)$ \\
\hline Data not available & $13(4.5)$ \\
\hline $4-7$ d & $70(24.4)$ \\
\hline $8-14$ d & $101(35.2)$ \\
\hline $15+$ days & $103(35.9)$ \\
\hline
\end{tabular}

$\overline{\mathrm{cm}}=$ centimeter; $\mathrm{CRF}=$ case report form; $\mathrm{IQR}=$ interquartile range;

$\mathrm{kg}=$ kilogram; $\mathrm{n}=$ number; $\mathrm{SD}=$ standard deviation.

${ }^{1}$ Data source: CRF.

${ }^{2}$ Data source: patient survey.

\section{Results}

There were 307 patients enrolled into the Electronic Data Capture (EDC) system. Twelve patients were excluded from the final analysis because they were entered into the system after the data entry cutoff date; three patients were found not to meet the eligibility criteria for failure on at least two prophylactic migraine treatments, three patients failed to complete the survey, and two patients were entered in duplicate. Overall, 287 migraine patients were included in the analysis.

The data presented hereafter describe the demographics and migraine-related clinical characteristics of the study population, including underlying comorbidities and migraine-related acute and prophylactic treatment history. We also report the humanistic burden of migraine and its impact on the QoL from the results of five validated PRO tools. Finally, the results presented here give a detailed overview of the economical burden associated with both episodic (low-frequency and high-frequency) and CM, including direct and indirect healthcare costs as well as indirect costs related to lost work productivity.

\section{Demographic and Clinical Characteristics}

Table 1 summarizes the key clinical and demographic characteristics of the patients' population. The mean age at enrollment was 46 years and $44.3 \%$ of the cohort was employed full-time. The mean time since migraine diagnosis was 14.3 years, while the mean time since first onset for migraine symptoms was 25.8 years. Patients' experienced a mean of 14.1 migraine days per month, over the past 3 months prior to study enrollment. High-frequency EM ( 8 to 14 MMDs) and CM (15 + MMDs) made up the majority of the cohort (35.2\% and $35.9 \%$, respectively).

Documented comorbidities among the study population are summarized in Table 2 . The majority of the study population (72.8\%) had underlying conditions, with the most commonly reported being depression $(26.3 \%)$.

\section{Patient-Reported Outcomes}

The humanistic burden of migraine was assessed through five validated PRO tools. Tables S1-S3 provide detailed summaries for each of the PRO assessments.

Based on the EQ-5D, $38.6 \%$ of patients reported moderate to severe problems performing usual activities. Moderate to severe 


\section{Table 2: Documented comorbidities of migraine patients in Canada $(n=287)$}

\begin{tabular}{|c|c|}
\hline Comorbidities & $N(\%)$ \\
\hline No comorbidities $^{1}$ & $78(27.2)$ \\
\hline Any comorbidity $^{1}$ & $209(72.8)$ \\
\hline \multicolumn{2}{|l|}{ Cardiovascular disorders } \\
\hline Cardiovascular disease & $6(2.9)$ \\
\hline Hyperlipidemia & $18(8.6)$ \\
\hline Hypertension & $24(11.5)$ \\
\hline Stroke & $0(0)$ \\
\hline \multicolumn{2}{|l|}{ Gastrointestinal disorders } \\
\hline Irritable bowel syndrome & $16(7.7)$ \\
\hline Gastrointestinal problems/dyspepsia & $26(12.4)$ \\
\hline \multicolumn{2}{|l|}{ Musculoskeletal/soft tissue disorders } \\
\hline Arthritis & $0(0)$ \\
\hline \multicolumn{2}{|l|}{ Neurological disorders } \\
\hline Epilepsy & $2(1.0)$ \\
\hline Multiple sclerosis & $0(0)$ \\
\hline Neuromuscular disorder & $2(1.0)$ \\
\hline Neuropathic pain & $3(1.4)$ \\
\hline Restless leg syndrome & $3(1.4)$ \\
\hline \multicolumn{2}{|l|}{ Pain disorders } \\
\hline Chronic back pain & $11(5.3)$ \\
\hline Fibromyalgia & $13(6.2)$ \\
\hline \multicolumn{2}{|l|}{ Psychiatric disorders } \\
\hline Anxiety/panic disorder & $28(13.4)$ \\
\hline Bipolar disorder & $3(1.4)$ \\
\hline Depression & $55(26.3)$ \\
\hline Psychosis/schizophrenia & $1(0.5)$ \\
\hline Substance abuse (alcohol/drugs) & $0(0)$ \\
\hline \multicolumn{2}{|l|}{ Respiratory disorders } \\
\hline Asthma/COPD & $26(12.4)$ \\
\hline \multicolumn{2}{|l|}{ Endocrine disorders } \\
\hline Diabetes & $8(3.8)$ \\
\hline Thyroid disease & $41(19.6)$ \\
\hline \multicolumn{2}{|l|}{ Other disorders } \\
\hline Obesity & $5(2.4)$ \\
\hline Sleep disorders & $23(11.0)$ \\
\hline Other & $85(40.7)$ \\
\hline
\end{tabular}

COPD $=$ chronic obstructive pulmonary disorder; $\mathrm{n}=$ number.

Data source: CRF.

${ }^{1}$ The results represent the full study population $(n=287)$.

pain/discomfort and anxiety/depression were reported by $62.7 \%$ and $38 \%$ of the patients, respectively. Median EuroQol Visual Analogue Scale (EQ VAS) was 65, where 0 refers to the worst health and 100 refers to the best health the respondent could imagine.

The HIT- $6^{\mathrm{TM}}$ and MIDAS tools were used to measure the impact of headaches on daily life (i.e. ability to function on the job, at school, at home, and in social situations). The mean HIT-6 ${ }^{\mathrm{TM}}$ score was 55.1 , with $50.2 \%$ of patients reporting some impact (score of 50-55), 29.6\% reporting substantial impact (score of 56-59), and $13.2 \%$ reporting severe impact (score of $60+$ ) on daily life. A MIDAS score greater than 20 indicates severe disability; the mean MIDAS score was reported as 81.4 and the median score was 48.0 among the study population. Specifically, 223 patients $(78 \%)$ indicated that they experienced severe disability (grade IV) on their daily life due to their headaches at the time of study enrollment.

WPAI outcomes are expressed as impairment percentages, with higher numbers indicating greater impairment and less productivity (i.e. worse outcomes). Survey respondents reported missing an average of $20.6 \%$ of their total work time due to migraine and experienced on average $47 \%$ impairment while working due to migraine. Furthermore, survey respondents reported an overall mean of $52.4 \%$ activity impairment due to migraine.

\section{Treatment History}

Treatment history prior to study enrollment is summarized in Table 3. Triptans were among the most commonly used treatments and multiple uses within the category were documented. The most commonly used triptan was rizatriptan, with nearly $50 \%$ of the study population reporting use. Among prophylactic treatments, topiramate was the most commonly used antiepileptic $(79.8 \%)$ and the most prevalent prophylactic treatment overall. History of onabotulinum toxin A (BoTN) use (60.6\%) was also among the most widely documented prophylactic migraine treatment among the study population, although CM patients represented only $35.9 \%$ of this study population. Interestingly, our data show that history of BoTN use was reported for both episodic and CM patients with a majority belonging to the chronic and high-frequency EM categories (data not shown). However, these data do not suggest an off-label use of BoTN because migraine classification of the study patient population was only based on the MMDs reported for the 3 months prior to enrollment, and these episodic patients would have had a history of CM prior to the initiation of BoTN.

Patients failed on average three therapies due to lack of efficacy. Prophylactic treatment failure on migraine medications received by patients during the 12 months prior to study enrollment is summarized in Table 4. Amitriptyline was the most used antidepressant $(21.6 \%)$ with a documented failure due to efficacy and tolerability of $14.5 \%$ and $12.9 \%$, respectively. In terms of the antiepileptic treatment category, topiramate use was documented in $32.1 \%$ of the study population and associated with a $17.4 \%$ and $18.5 \%$ efficacy and tolerability failure, respectively. For antihypertensive treatments, propranolol use was documented in $9.1 \%$ of the population and among users, $15.4 \%$ had efficacy failure and $19.2 \%$ experienced tolerability failure. Erenumab (54.4\%) and BoTN $(34.8 \%)$ were among the most prevalent prophylactic treatments and associated with an efficacy failure of $9.0 \%$ and $14.0 \%$, respectively. A $3.8 \%$ tolerability failure was noted for erenumab, while BoTN had none.

\section{Resource Utilization and Economic Burden}

Although the majority of the study population had underlying conditions, only direct and indirect costs related to their migraine 


\section{Table 3: Treatment history prior to study enrollment, among migraine patients in Canada $(n=287)$}

\begin{tabular}{|c|c|}
\hline Treatment & $N(\%)$ \\
\hline No prior treatment & $1(0.3)$ \\
\hline Any prior treatment & $286(99.7)$ \\
\hline \multicolumn{2}{|l|}{ Acute treatments } \\
\hline \multicolumn{2}{|l|}{ Triptans } \\
\hline Almotriptan & $123(42.9)$ \\
\hline Eletriptan & $98(34.1)$ \\
\hline Frovatriptan & $43(15)$ \\
\hline Naratriptan & $51(17.8)$ \\
\hline Rizatriptan & $142(49.5)$ \\
\hline Sumatriptan & $130(45.3)$ \\
\hline Zolmitriptan & $126(43.9)$ \\
\hline Acetaminophen & $126(43.9)$ \\
\hline \multicolumn{2}{|l|}{ NSAIDs } \\
\hline Acetylsalicylic acid (ASA) & $30(10.5)$ \\
\hline Ibuprofen & $149(51.9)$ \\
\hline Naproxen sodium & $153(53.3)$ \\
\hline Diclofenac tablets & $37(12.9)$ \\
\hline Diclofenac powder for oral solution & $98(34.1)$ \\
\hline Ketorolac & $46(16)$ \\
\hline \multicolumn{2}{|l|}{ Ergots } \\
\hline Dihydroergotamine & $42(14.6)$ \\
\hline \multicolumn{2}{|l|}{ Anti-nausea medications } \\
\hline Metoclopramide & $63(22)$ \\
\hline Domperidone & $7(2.4)$ \\
\hline Ondansetron hydrochloride (Zofran $\left.{ }^{\oplus}\right)$ & $16(5.6)$ \\
\hline Opioids & $61(21.3)$ \\
\hline $\begin{array}{l}\text { Combination analgesics with opioids } \\
\text { or barbiturates }\end{array}$ & $96(33.4)$ \\
\hline $\begin{array}{l}\text { Combination analgesics without } \\
\text { opioids or barbiturates }\end{array}$ & $18(6.3)$ \\
\hline Other acute treatments & $92(32.1)$ \\
\hline \multicolumn{2}{|l|}{ Prophylactic treatments } \\
\hline \multicolumn{2}{|l|}{ Antidepressants } \\
\hline Amitriptyline & $213(74.2)$ \\
\hline Duloxetine (Cymbalta $\left.{ }^{\oplus}\right)$ & $35(12.2)$ \\
\hline Nortriptyline & $69(24)$ \\
\hline Venlafaxine & $45(15.7)$ \\
\hline \multicolumn{2}{|l|}{ Antiepileptics/anticonvulsants } \\
\hline $\begin{array}{l}\text { Divalproex sodium/valproic acid/ } \\
\text { sodium valproate }\end{array}$ & $51(17.8)$ \\
\hline Gabapentin (Neurontin ${ }^{\circledast}$ ) & $83(28.9)$ \\
\hline Topiramate $\left(\right.$ Topamax $\left.{ }^{\oplus}\right)$ & $229(79.8)$ \\
\hline \multicolumn{2}{|l|}{ Antihypertensives (beta-blockers) } \\
\hline Propanolol (Inderal ${ }^{\oplus}$ ) & $117(40.8)$ \\
\hline Timolol (Blocadren ${ }^{\circledR}$ ) & $1(0.3)$ \\
\hline Atenolol & $10(3.5)$ \\
\hline
\end{tabular}

Table 3: (Continued)

\begin{tabular}{|c|c|}
\hline Treatment & $N(\%)$ \\
\hline Metoprolol & $29(10.1)$ \\
\hline Nadolol & $44(15.3)$ \\
\hline \multicolumn{2}{|l|}{ Antihypertensives (ACEIs/ARBs) } \\
\hline${\text { Lisinopril (Zestril }{ }^{\circledR} \text { ) }}$ & $2(0.7)$ \\
\hline Candesartan & $80(27.9)$ \\
\hline \multicolumn{2}{|l|}{ Calcium channel blockers } \\
\hline Verapamil $\left(\right.$ Calan $\left.^{\circledR}\right)$ & $17(5.9)$ \\
\hline Flunarizine & $26(9.1)$ \\
\hline \multicolumn{2}{|l|}{ Serotonin antagonists } \\
\hline 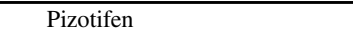 & $36(12.5)$ \\
\hline Onabotulinum toxin A (botox) & $174(60.6)$ \\
\hline \multicolumn{2}{|l|}{ Supplements } \\
\hline Magnesium & $76(26.5)$ \\
\hline Riboflavin & $56(19.5)$ \\
\hline Co-enzyme Q10 & $25(8.7)$ \\
\hline Other prophylactic treatments & $169(58.9)$ \\
\hline
\end{tabular}

$\overline{\mathrm{ACEI}}=$ angiotensin-converting enzyme inhibitor; $\mathrm{ARB}=$ angiotensin II receptor blockers; $\mathrm{n}=$ number.

Data source: CRF.

have been taken into consideration for the overall assessment of the burden of this disease.

Utilization and costs of prescribed migraine treatments from the 12 months prior to study enrollment are summarized in Table 5. Among acute treatments, ondansetron hydrochloride (\$415.93) and frovatriptan (\$188.75) were associated with the highest mean per patient monthly cost. Triptans incurred the highest annual cost when factoring in duration of use. Sumatrip$\tan (\$ 1,428.63)$, eletriptan $(\$ 1,251.08)$, and frovatriptan $(\$ 1,085.12)$ were associated with the largest mean annual costs for acute migraine medications.

Erenumab and BoTN were associated with the highest overall costs among prophylactic migraine treatments (monthly and annually). The mean annual per patient cost for BoTN was $\$ 2,443.71$. Since study enrollment coincided with the product launch of erenumab in Canada, the annual treatment duration could not be calculated for this treatment since the reported mean treatment duration was only of 3 months (i.e. the first 3 months post-erenumab launch in Canada). Therefore, the cost estimate of erenumab of $\$ 1,786.86$ shown in Table 5 reflects a mean treatment duration of 3 months only. Costs related to other prophylactic treatment classes were lower.

Annual frequency and costs associated with healthcare provider (HCP) visits over the 12 months prior to study enrollment are summarized in Table 6. The majority of the study population $(97.9 \%)$ visited a neurologist or headache specialist at least once, with a mean frequency of approximately five times per year. Primary care physicians were seen by $56.4 \%$ of the study population on an average of eight times per year. The highest mean annual frequencies of patient visits were observed for psychologist (18.9 visits), physiotherapist (18.6 visits), and chiropractors (16.7 visits), with these services being utilized by $9.1 \%, 15.3 \%$, and $18.8 \%$ of the 


\section{Table 4: Treatment failure on prophylactic migraine medications received by patients during the 12 months prior to study enrollment $(n=287)$}

\begin{tabular}{|c|c|c|c|c|c|c|c|}
\hline \multirow[b]{2}{*}{ Treatment } & \multirow[b]{2}{*}{$\begin{array}{c}\text { Patients on treatment, } \\
n(\%)^{1}\end{array}$} & \multirow[b]{2}{*}{$\begin{array}{c}\text { No treatment failure, } n \\
(\%)^{2}\end{array}$} & \multirow[b]{2}{*}{$\begin{array}{c}\text { Any treatment failure, } \\
n(\%)^{2}\end{array}$} & \multicolumn{4}{|c|}{ Type of treatment failure } \\
\hline & & & & $\begin{array}{c}\text { Efficacy failure, } \\
n(\%)^{2}\end{array}$ & $\begin{array}{c}\text { Tolerability failure, } \\
n(\%)^{2}\end{array}$ & $\begin{array}{c}\text { Other failure } \\
\text { (e.g. not suitable), } \\
n(\%)^{2}\end{array}$ & Unknown, $n(\%)^{2}$ \\
\hline \multicolumn{8}{|c|}{ Prophylactic treatments $(n=264)$} \\
\hline \multicolumn{8}{|c|}{ Antidepressants $(n=98)$} \\
\hline Amitriptyline & $62(21.6)$ & $31(50.0)$ & $31(50.0)$ & $9(14.5)$ & $8(12.9)$ & $0(0)$ & $14(22.6)$ \\
\hline $\begin{array}{l}\text { Duloxetine } \\
\text { (Cymbalta) }\end{array}$ & $10(3.5)$ & $4(40.0)$ & $6(60.0)$ & $0(0)$ & $0(0)$ & $0(0)$ & $6(60.0)$ \\
\hline Nortriptyline & $23(8.0)$ & $14(60.9)$ & $10(43.5)$ & $3(13.0)$ & $2(8.7)$ & $0(0)$ & $5(21.7)$ \\
\hline Venlafaxine & $18(6.3)$ & $11(61.1)$ & $7(38.9)$ & $1(5.6)$ & $0(0)$ & $0(0)$ & $6(33.3)$ \\
\hline \multicolumn{8}{|c|}{ Antiepileptics/anticonvulsants $(n=119)$} \\
\hline $\begin{array}{l}\text { Divalproex sodium/ } \\
\text { valproic acid/ } \\
\text { sodium valproate }\end{array}$ & $10(3.5)$ & $6(60.0)$ & $4(40.0)$ & $1(10.0)$ & $1(10.0)$ & $0(0)$ & $2(20.0)$ \\
\hline $\begin{array}{l}\text { Gabapentin } \\
\text { (Neurontin) }\end{array}$ & $27(9.4)$ & $13(48.1)$ & $15(55.6)$ & $5(18.5)$ & $1(3.7)$ & $0(0)$ & $9(33.3)$ \\
\hline $\begin{array}{l}\text { Topiramate } \\
\text { (Topamax) }\end{array}$ & $92(32.1)$ & $40(43.5)$ & $54(58.7)$ & $16(17.4)$ & $17(18.5)$ & $0(0)$ & $21(22.8)$ \\
\hline \multicolumn{8}{|c|}{ Antihypertensives (beta-blockers) $(n=56)$} \\
\hline Propanolol (Inderal) & $26(9.1)$ & $12(46.2)$ & $14(53.8)$ & $4(15.4)$ & $5(19.2)$ & $0(0)$ & $5(19.2)$ \\
\hline Timolol (Blocadren) & $0(0)$ & $0(0)$ & $0(0)$ & $0(0)$ & $0(0)$ & $0(0)$ & $0(0)$ \\
\hline Atenolol & $1(0.3)$ & $1(100.0)$ & $0(0)$ & $0(0)$ & $0(0)$ & $0(0)$ & $0(0)$ \\
\hline Metoprolol & $17(5.9)$ & $10(58.8)$ & $7(41.2)$ & $1(5.9)$ & $1(5.9)$ & $0(0)$ & $5(29.4)$ \\
\hline Nadolol & $13(4.5)$ & $9(69.2)$ & $4(30.8)$ & $2(15.4)$ & $0(0)$ & $0(0)$ & $2(15.4)$ \\
\hline \multicolumn{8}{|l|}{ ACEIs/ARBs $(n=36)$} \\
\hline Lisinopril (Zestril) & $0(0)$ & $0(0)$ & $0(0)$ & $0(0)$ & $0(0)$ & $0(0)$ & $0(0)$ \\
\hline Candesartan & $36(12.5)$ & $22(61.1)$ & $14(38.9)$ & $8(22.2)$ & $3(8.3)$ & $0(0)$ & $3(8.3)$ \\
\hline \multicolumn{8}{|c|}{ Calcium channel blockers $(n=5)$} \\
\hline Verapamil (Calan) & $3(1.0)$ & $2(66.7)$ & $1(33.3)$ & $1(33.3)$ & $0(0)$ & $0(0)$ & $0(0)$ \\
\hline Flunarizine & $2(0.7)$ & $1(50.0)$ & $1(50.0)$ & $0(0)$ & $0(0)$ & $0(0)$ & $1(50.0)$ \\
\hline \multicolumn{8}{|c|}{ Serotonin antagonists $(n=5)$} \\
\hline Pizotifen & $5(1.7)$ & $3(60.0)$ & $2(40.0)$ & $1(20.0)$ & $0(0)$ & $0(0)$ & $1(20.0)$ \\
\hline $\begin{array}{l}\text { Onabotulinum toxin } \\
\text { A (botox) }\end{array}$ & $100(34.8)$ & $72(72.0)$ & $29(29.0)$ & $14(14.0)$ & $0(0)$ & $0(0)$ & $15(15.0)$ \\
\hline \multicolumn{8}{|l|}{ Supplements $(n=43)$} \\
\hline Magnesium & 34 (11.8) & $22(64.7)$ & $12(35.3)$ & $5(14.7)$ & $0(0)$ & $0(0)$ & $7(20.6)$ \\
\hline Riboflavin & $18(6.3)$ & $9(50.0)$ & $9(50.0)$ & $3(16.7)$ & $0(0)$ & $0(0)$ & $6(33.3)$ \\
\hline Co-enzyme Q10 & $6(2.1)$ & $4(66.7)$ & $2(33.3)$ & $0(0)$ & $0(0)$ & $0(0)$ & $2(33.3)$ \\
\hline \multicolumn{8}{|l|}{ Other $(n=205)$} \\
\hline $\begin{array}{l}\text { Erenumab } \\
\quad(\text { Aimovig } \\
\end{array}$ & $156(54.4)$ & $108(69.2)$ & $51(32.7)$ & $14(9.0)$ & $6(3.8)$ & $0(0)$ & $31(19.9)$ \\
\hline
\end{tabular}

ACEI = angiotensin-converting enzyme inhibitor; ARB = angiotensin II receptor blockers; $\mathrm{n}=$ number.

${ }^{1}$ The denominator for calculating the percentage of patients on each treatment will be total study sample.

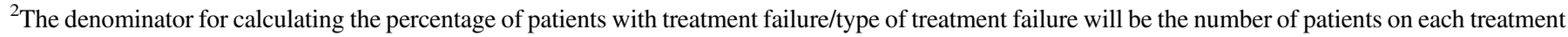
type (i.e. number of patients presented in the first column of the table).

Treatment category n's represent the number of patients who took at least one drug in that associated treatment class.

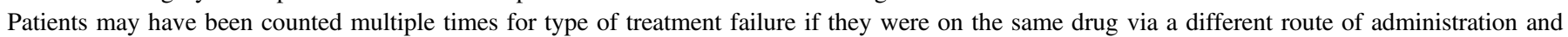

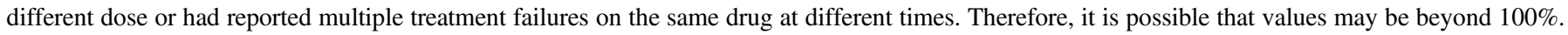
This affected less than $5 \%$ of the study population.

Sums may not add up to $100 \%$ since treatments are not mutually exclusive.

Data source: CRF. 
Table 5: Utilization and costs of treatments prescribed to migraine patients over the 12 months prior to study enrollment $(n=287)$

\begin{tabular}{|c|c|c|c|c|c|c|c|}
\hline \multirow[b]{2}{*}{ Treatment } & \multirow[b]{2}{*}{ All Patients, n (\%) } & \multicolumn{2}{|c|}{ Monthly cost in \$CAD per patient ${ }^{1}$} & \multicolumn{2}{|c|}{ Duration of use (months) } & \multicolumn{2}{|c|}{ Annual cost per duration of use $(\$ C A D)^{2,3}$} \\
\hline & & Mean (SD) & Median (IQR) & Mean (SD) & Median (IQR) & Mean (SD) & Median (IQR) \\
\hline \multicolumn{8}{|l|}{ Acute treatments ${ }^{4}$} \\
\hline \multicolumn{8}{|l|}{ Triptans $(n=230)$} \\
\hline Almotriptan & $57(19.9)$ & $137.15(81.47)$ & $107.58(78.24-166.26)$ & $12(15.6)$ & $5(1.8-12.0)$ & $779.58(1,015.00)$ & $469.44(304.98-821.52)$ \\
\hline Eletriptan & $42(14.6)$ & $149.59(88.12)$ & $126.13(70.63-201.80)$ & $18.7(16.0)$ & $19.5(5.2-32.1)$ & $1,251.08(1,096.90)$ & \begin{tabular}{|l}
$1,089.72(432.94-2,069.21)$ \\
\end{tabular} \\
\hline Frovatriptan & $10(3.5)$ & $188.75(114.78)$ & $175.00(87.50-250.00)$ & 6.7 (NA) & 6.7 (NA) & $1,085.12(\mathrm{NA})$ & $1,085.12$ (NA) \\
\hline Naratriptan & $20(7.0)$ & $122.56(68.25)$ & $109.04(69.79-157.02)$ & $16.6(25.5)$ & $5.5(1.1-32.1)$ & $742.13(694.30)$ & $604.48(195.41-1,288.84)$ \\
\hline Rizatriptan & $63(22.0)$ & $161.35(91.71)$ & $135.73(90.49-226.22)$ & $8.3(7.3)$ & $6.4(3.3-13.4)$ & $962.1(259.00)$ & \begin{tabular}{|l|l}
$1,000.87(766.47-1,157.74)$ \\
\end{tabular} \\
\hline Sumatriptan & $72(25.1)$ & $142.21(87.52)$ & $113.69(82.69-191.21)$ & $58.5(102.2)$ & $18.3(4.8-27.4)$ & $1,428.63(643.60)$ & $1,244.88(1,054.25-1,803.02)$ \\
\hline Zolmitriptan & $68(23.7)$ & $96.33(63.77)$ & 73.68 (44.21-121.57) & $12.6(21.9)$ & $3.7(1-12)$ & $343.21(284.60)$ & $298.6(225.41-373.25)$ \\
\hline Acetaminophen & $48(16.7)$ & $0.83(0.56)$ & $0.66(0.42-1.26)$ & $5.4(7.7)$ & $5.4(0-10.9)$ & $7.84(\mathrm{NA})$ & $7.84(\mathrm{NA})$ \\
\hline \multicolumn{8}{|l|}{ NSAIDs $(n=151)$} \\
\hline $\begin{array}{l}\text { Acetylsalicylic acid } \\
\text { (ASA) }\end{array}$ & $0(0)$ & - & - & - & - & - & - \\
\hline Ibuprofen & $49(17.1)$ & $2.32(1.44)$ & $1.98(1.44-2.70)$ & - & - & - & - \\
\hline Naproxen sodium & $57(19.9)$ & $4.15(2.59)$ & $3.30(2.64-4.95)$ & $29.1(27.7)$ & $22.6(7.9-43)$ & $34.58(12.00)$ & $35.64(28.54-43.56)$ \\
\hline Diclofenac tablets & $18(6.3)$ & $3.71(1.91)$ & $2.75(2.25-50.00)$ & - & - & - & - \\
\hline $\begin{array}{l}\text { Diclofenac powder for } \\
\text { oral solution }\end{array}$ & $67(23.3)$ & $10.59(6.36)$ & $8.58(5.46-13.26)$ & $15.3(15.4)$ & $10.1(4.9-25.7)$ & $59.52(48.30)$ & $39.21(30.12-88.92)$ \\
\hline Ketorolac & $19(6.6)$ & $13.88(7.46)$ & $13.87(7.30-21.90)$ & $0.2(0)$ & $0.2(0.2-0.2)$ & $2.34(0.10)$ & $2.34(2.28-2.40)$ \\
\hline \multicolumn{8}{|l|}{ Ergots $(n=47)$} \\
\hline Dihydroergotamine & $13(4.5)$ & $148.19(86.11)$ & $122.22(78.57-253.17)$ & $1(1.7)$ & $0.3(0.2-0.5)$ & $151.48(139.70)$ & $114.29(68.92-234.04)$ \\
\hline Metoclopramide & $29(10.1)$ & $1.00(0.61)$ & $0.84(0.56-1.33)$ & $0.1(0.1)$ & $0.1(0-0.2)$ & $0.25(0)$ & $0.25(0.22-0.27)$ \\
\hline Domperidone & $4(1.4)$ & $0.5(0.12)$ & $0.54(0.42-0.58)$ & - & - & - & - \\
\hline $\begin{array}{l}\text { Ondansetron } \\
\text { hydrochloride (Zofran) }\end{array}$ & $12(4.2)$ & $415.93(175.93)$ & $501.65(255.56-567.90)$ & $0.4(\mathrm{NA})$ & $0.4(0.4-0.4)$ & $121.43(\mathrm{NA})$ & $121.43(\mathrm{NA})$ \\
\hline Opioids & $21(7.3)$ & $17.29(9.34)$ & $15.08(9.28-23.20)$ & 0.1 (NA) & $0.1(0.1-0.1)$ & - & - \\
\hline $\begin{array}{l}\text { Combination analgesics } \\
\text { with opioids or } \\
\text { barbiturates }\end{array}$ & $27(9.4)$ & $2.36(1.23)$ & $1.96(1.54-3.50)$ & $43.6(\mathrm{NA})$ & $43.6(43.6-43.6)$ & $23.52(\mathrm{NA})$ & $23.52(23.52-23.52)$ \\
\hline $\begin{array}{l}\text { Combination analgesics } \\
\text { without opioids or } \\
\text { barbiturates }\end{array}$ & $5(1.7)$ & $0(0)$ & $0(0-0)$ & - & - & - & - \\
\hline
\end{tabular}

Prophylactic treatments

Antidepressants ( $n=98)$

\begin{tabular}{|c|c|c|c|c|c|c|c|}
\hline Amitriptyline & $62(21.6)$ & $2.40(0)$ & $2.40(2.40-2.40)$ & $19.3(25.2)$ & $11.8(6.0-17.8)$ & $20.87(10.10)$ & $27.03(14.41-28.80)$ \\
\hline Duloxetine (Cymbalta) & $10(3.5)$ & $21.90(0)$ & $21.90(21.90-21.90)$ & - & - & - & - \\
\hline Nortriptyline & $23(8.0)$ & $11.40(0)$ & $11.40(11.40-11.40)$ & $8.5(9.6)$ & $7.9(0.8-9.7)$ & $69.66(61.00)$ & $89.63(9.38-111.00)$ \\
\hline Venlafaxine & $18(6.3)$ & $5.10(0)$ & $5.10(5.10-5.10)$ & $6.1(1.3)$ & $6.1(5.2-7.0)$ & $31.12(6.80)$ & $31.12(26.34-35.90)$ \\
\hline
\end{tabular}




\begin{tabular}{|c|c|c|c|c|c|c|c|}
\hline \multirow[b]{2}{*}{ Treatment } & \multirow[b]{2}{*}{ All Patients, n (\%) } & \multicolumn{2}{|c|}{ Monthly cost in \$CAD per patient ${ }^{1}$} & \multicolumn{2}{|c|}{ Duration of use (months) } & \multicolumn{2}{|c|}{ Annual cost per duration of use (\$CAD) $)^{2,3}$} \\
\hline & & Mean (SD) & Median (IQR) & Mean (SD) & Median (IQR) & Mean (SD) & Median (IQR) \\
\hline \multicolumn{8}{|c|}{ Antiepileptics/anticonvulsants $(n=120)$} \\
\hline $\begin{array}{l}\text { Divalproex sodium/ } \\
\text { valproic acid/sodium } \\
\text { valproate }\end{array}$ & $10(3.5)$ & $10.50(0)$ & $10.50(10.50-10.50)$ & $38.7(50.6)$ & $38.7(2.9-74.5)$ & $78.37(67.40)$ & $78.37(30.74-126.00)$ \\
\hline Gabapentin (Neurontin) & $27(9.4)$ & $42.00(0)$ & $42.00(42.00-42.00)$ & $25.2(23.1)$ & $18.1(6.4-51.0)$ & $425.80(135.40)$ & $504.00(269.41-504.00)$ \\
\hline Topiramate (Topamax) & $92(32.1)$ & $16.50(0)$ & $16.50(16.50-16.50)$ & $18.6(23.6)$ & $10.1(4.0-16.4)$ & 132.94 (74.70) & $167.17(65.40-198.00)$ \\
\hline \multicolumn{8}{|c|}{ Antihypertensives (beta-blockers) $(n=56)$} \\
\hline Propanolol (Inderal) & $26(9.1)$ & $6.60(0)$ & $6.60(6.60-6.60)$ & $14(21.9)$ & $2.8(2.0-20.9)$ & $37.64(30.90)$ & $18.67(13.24-79.20)$ \\
\hline Timolol (Blocadren) & $0(0)$ & - & - & - & - & - & - \\
\hline Atenolol & $1(0.3)$ & $3.30(\mathrm{NA})$ & 3.30 (NA) & - & - & - & - \\
\hline Metoprolol & $17(5.9)$ & $3.60(0)$ & $3.60(3.60-3.60)$ & $30.7(29.8)$ & $30.7(9.6-51.8)$ & $38.89(6.10)$ & $38.89(34.58-43.20)$ \\
\hline Nadolol & $13(4.5)$ & $15.00(0)$ & $15.00(15.00-15.00)$ & $12(6.2)$ & $12(7.6-16.4)$ & 146.99 (46.70) & $146.99(113.98-180.00)$ \\
\hline \multicolumn{8}{|c|}{ Antihypertensives (ACEIs/ARBs) $(n=36)$} \\
\hline Lisinopril (Zestril) & $0(0)$ & - & - & - & - & - & - \\
\hline Candesartan & $36(12.5)$ & $6.60(0)$ & $6.60(6.60-6.60)$ & $2.3(1.9)$ & $2.8(0.2-3.9)$ & $15.20(12.80)$ & $18.45(1.09-26.05)$ \\
\hline \multicolumn{8}{|c|}{ Calcium channel blockers $(n=5)$} \\
\hline Verapamil (Calan) & $3(1.0)$ & $16.20(0)$ & $16.20(16.20-16.20)$ & - & - & - & - \\
\hline Flunarizine & $2(0.7)$ & $21.90(0)$ & $21.90(21.90-21.90)$ & $3.2(\mathrm{NA})$ & $3.2(\mathrm{NA})$ & 70.60 (NA) & 70.60 (NA) \\
\hline \multicolumn{8}{|l|}{ Serotonin antagonists } \\
\hline $\begin{array}{l}\text { Pizotifen } \\
\end{array}$ & $5(1.7)$ & $25.20(0)$ & $25.20(25.20-25.20)$ & $\overline{-}$ & $\overline{-}$ & $\overline{-}$ & - \\
\hline $\begin{array}{l}\text { Onabotulinum toxin A } \\
\text { (Botox) }^{5}\end{array}$ & $100(34.8)$ & $252.10(0)$ & $252.10(252.10-252.10)$ & $27.1(29.9)$ & $17.5(7.0-26.0)$ & $2,443.71(833.00)$ & $3,025.20(1,758.07-3,025.20)$ \\
\hline \multicolumn{8}{|l|}{ Supplements $(n=43)$} \\
\hline Magnesium & $34(11.8)$ & $4.20(0)$ & $4.20(4.20-4.20)$ & $10.6(11.6)$ & $4.8(3.0-23.9)$ & $27.71(20.00)$ & $20.17(12.57-50.40)$ \\
\hline Riboflavin $^{6}$ & $18(6.3)$ & $3.30(0)$ & $3.30(3.30-3.30)$ & $0.7(\mathrm{NA})$ & 0.7 (NA) & 2.17 (NA) & 2.17 (NA) \\
\hline Co-enzyme Q10 & $6(2.1)$ & $2.70(0)$ & 2.70 (NA) & - & - & - & - \\
\hline \multicolumn{8}{|l|}{ Other } \\
\hline Erenumab (Aimovig ${ }^{\circledR}$ ) & $156(54.4)$ & $532.00(0)$ & $532.00(532.00-532.00)$ & $3(1.7)$ & $2.9(1.9-4.6)$ & $1,786.86(709.07)$ & $1,619.94(1,223.60-2,537.64)$ \\
\hline
\end{tabular}

ACEI = angiotensin-converting enzyme inhibitor; ARB = angiotensin II receptor blockers; $\mathrm{n}=$ number; $=$ not reported; NA = not applicable due to insufficient data

${ }^{1}$ Per patient with any utilization of the therapy.

${ }^{2}$ Calculated based on drug dose and frequency information from CRF Question 13, and the unit costs for each medication. Annual cost per treatment is the product of duration of use and total cost of treatment, where the cost is calculated based on frequency and amount of drug use.

${ }^{3}$ Annual cost per treatment is the product of duration of use within the past 12 months and total cost of treatment, where the cost is weighted average calculated based on standard unit costs, doses, and frequency of use.

${ }^{4}$ Frequency of drug use was calculated using the average number of migraine days.

${ }^{5}$ The cost of botox only and does not include the injection procedure cost.

${ }^{6}$ Riboflavin use was reported by only one patient and for 20 days. Hence, the monthly cost, which is based on number of migraine days, is more than annual cost that is calculated using actual yearly duration of use.

Treatment category n's represent the number of patients who took at least one drug in that associated treatment class.

Drug unit costs were standardized using a weighted average for dosages as reported in the formulary and a mean daily cost was then applied based on reported dose and frequency of use. It was also assumed that prophylactic drugs were taken for 30 days each month. Thus, there is limited dispersion of monthly costs SD and IQR.

Data source: CRF; unit costs. 
Table 6: Frequency and costs of healthcare provider visits for migraine patients in Canada, over the 12 months prior to study enrollment $(n=287)$

\begin{tabular}{|c|c|c|c|}
\hline Healthcare providers & Patients with any utilization ${ }^{1}, n(\%)$ & Annual frequency ${ }^{1,2,3}$, mean (SD) & $\begin{array}{c}\text { Annual cost in \$CAD per patient }{ }^{1,2} \text {, mean } \\
\text { (SD) }\end{array}$ \\
\hline Neurologist/headache specialist & $281(97.9)$ & $4.5(3.6)$ & $812.40(652.00)$ \\
\hline Nurse practitioner/physician assistant & $21(7.3)$ & $8.8(14.9)$ & $321.99(546.80)$ \\
\hline Ophthalmologist & $22(7.7)$ & $3.1(2.5)$ & $262.57(207.90)$ \\
\hline Psychiatrist & $23(8.0)$ & $7.4(8.2)$ & $1,529.02(1,693.00)$ \\
\hline Primary care physician/family doctor & $162(56.4)$ & $8.3(11.0)$ & $659.43(880.20)$ \\
\hline Psychologist & $26(9.1)$ & $18.9(21.7)$ & $1,225.65(1,406.80)$ \\
\hline Acupuncturist & $24(8.4)$ & $15.3(15.9)$ & $794.30(827.20)$ \\
\hline Physiotherapist & $44(15.3)$ & $18.6(18.8)$ & $2,038.77(2,068.40)$ \\
\hline Chiropractor & $54(18.8)$ & $16.7(14.1)$ & $1,219.56(1,026.90)$ \\
\hline Homeopath & $8(2.8)$ & $2.5(5.6)$ & $374.25(843.00)$ \\
\hline Massage therapist & $83(28.9)$ & $13.3(14.1)$ & $1,324.18(1,405.30)$ \\
\hline Naturopathic doctor & $10(3.5)$ & $6.4(12.3)$ & $1,248(2,388.60)$ \\
\hline Osteopath & $30(10.5)$ & $11.7(10.7)$ & $937.93(859.20)$ \\
\hline Other & $49(17.1)$ & $9.7(15.9)$ & NA \\
\hline
\end{tabular}

CAD = Canadian dollar; $\mathrm{n}=$ number; NA = not applicable; SD = standard deviation.

${ }^{1}$ For each healthcare provider type, if visits to the provider are reported in both the CRF and patient survey, the CRF data will be used to avoid doublecounting.

${ }^{2}$ Per patient with any utilization of the therapy.

${ }^{3}$ Three-month data from the patient survey will be extrapolated to 1 year, to estimate annual utilization and costs.

Data source: CRF, patient survey.

study population, respectively. The calculated annual cost per patient was highest for physiotherapist visits $(\$ 2,038.77)$, followed by psychiatrist visits $(\$ 1,529.02)$ and massage therapist visits $(\$ 1,324.18)$. The primary cost driver was associated with frequency of visits.

Frequency and costs for outpatient tests and procedures are presented in Table 7. BoTN injections were the highest utilized nonsurgical procedure among the study population $(31.4 \%)$ and were used on average four times per year, resulting in an annual cost of $\$ 440.60$ per patient (cost of administration only). However, the highest annual costs were associated with occipital nerve blocks (utilized by $18.1 \%$ of patients) and MRI scans (utilized by $11.1 \%$ of patients).

Less than five percent of the study population reported use of a device with a mean of 19 MMDs and 70\% of them diagnosed with CM (data not shown). Among those who reported, Cefaly ${ }^{\circledR}$ and $\mathrm{GammaCore}^{\circledR}$ were the most frequently used $(4.9 \%$ and $4.5 \%$, respectively) and GammaCore ${ }^{\circledR}$ incurred the highest costs $(\$ 1,007.35)$. The "Other" category consisted of various reported items such as Aculief Wearable Acupressure ${ }^{\mathrm{TM}}$, Magic Bag ${ }^{\circledR}$, dental night guards, and vibrating heat pads.

Indirect costs associated with loss of productivity are summarized in Table 8. Of the survey respondents, $61.3 \%$ of patients reported an average of 60.6 days in which productivity was reduced by half or more due to migraine, $61.0 \%$ patients reported missed time from work, and $15.7 \%$ of the respondents reported unemployment due to migraine for an average length of 220 days. The loss productivity, missed time from work, and unemployment due to migraine were associated with a mean annual costs of $\$ 12,462.87$, $\$ 10,458.08$, and $\$ 16,525.20$, respectively. Long-term disability associated with migraine incurred the highest mean annual cost at $\$ 32,679.34$. Expectedly, overall estimates of per patient annual indirect costs were reduced when considering the entire study sample (i.e. assuming a cost of zero dollars for those who did not report lost productivity).

Total annual direct and indirect costs are summarized by migraine type in Table 9. Total annual per patient costs for low-frequency EM were lowest $(\$ 15,651.34)$, followed by highfrequency EM $(\$ 24,884.79)$ and CM $(\$ 25,668.89)$ types. Direct non-healthcare costs and indirect costs were observed to be key drivers for increasing the overall cost of high-frequency EM closer towards that of CM. Furthermore, indirect costs were associated with the highest overall cost category across each subgroup and in the overall study population. Considering the entire study population (low-frequency EM, high-frequency EM, and CM patients), the total annual cost associated with migraine in Canada was estimated to be $\$ 23,756.04$ per patient (data not shown).

\section{Discussion}

While previous studies have estimated the burden of migraine in Canada, ${ }^{4,8,11}$ these studies focused particularly on the direct healthcare cost component of the economic burden of migraine. There is less published Canadian data available on the economic burden and cost related to disability due to migraine and its impact on work productivity. Consequently, this study provides 
Table 7: Frequency and costs of outpatient tests, procedures, and non-pharmacologic interventions for migraine patients in Canada, over the 12 months prior to study enrollment $(n=287)$

\begin{tabular}{|c|c|c|c|}
\hline & Patients with any utilization $^{1} n(\%)$ & Annual frequency ${ }^{1}$, mean (SD) & $\begin{array}{c}\text { Annual cost in \$CAD per patient }{ }^{1} \text {, mean } \\
\text { (SD) }\end{array}$ \\
\hline \multicolumn{4}{|l|}{ Laboratory tests } \\
\hline Blood test & $93(32.4)$ & $1.7(1.7)$ & $51.90(51.90)$ \\
\hline \multicolumn{4}{|l|}{ Nonsurgical procedures/interventions } \\
\hline Head scan - MRI & $32(11.1)$ & $1.0(0.2)$ & $796.90(136.60)$ \\
\hline Head scan - CT scan & $10(3.5)$ & $1.0(0)$ & $341.70(0)$ \\
\hline Spinal tap (lumbar puncture) & $1(0.3)$ & $1.0(\mathrm{NA})$ & 69.80 (NA) \\
\hline EEG & $5(1.7)$ & $1.0(0)$ & $49.10(0)$ \\
\hline Eye exam & $56(19.5)$ & $1.2(0.7)$ & $63.40(37.20)$ \\
\hline TENS & $1(0.3)$ & 1.0 (NA) & 109.80 (NA) \\
\hline 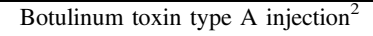 & $90(31.4)$ & $3.5(1.9)$ & $440.60(241.20)$ \\
\hline Occipital nerve block & $52(18.1)$ & $7.5(5.2)$ & $732.30(511.20)$ \\
\hline
\end{tabular}

CAD = Canadian dollar; $\mathrm{n}=$ number; SD = standard deviation.

${ }^{1}$ Per patient with any utilization of the therapy.

${ }^{2}$ The cost of injection procedure only.

Data source: CRF.

Table 8: Indirect costs among migraine patients in Canada, over the 12 months prior to study enrollment $(n=287)$

\begin{tabular}{|c|c|c|c|c|c|c|}
\hline \multirow[b]{2}{*}{ Indirect cost category } & \multirow[b]{2}{*}{$n(\%)$} & \multirow{2}{*}{$\begin{array}{c}\begin{array}{c}\text { Number of } \\
\text { days per } \\
\text { year }\end{array} \\
\text { Mean (SD) }\end{array}$} & \multicolumn{2}{|c|}{$\begin{array}{l}\text { Annual cost per patient with } \\
\text { any utilization } \\
(\$ C A D)\end{array}$} & \multicolumn{2}{|c|}{$\begin{array}{c}\text { Overall annual cost per } \\
\text { migraine patient }^{1} \\
(\$ C A D)\end{array}$} \\
\hline & & & Mean (SD) & Median (IQR) & Mean (SD) & Median (IQR) \\
\hline \multicolumn{7}{|l|}{ Lost productivity } \\
\hline Missed time from work & $175(61.0)$ & $46.0(84.6)$ & $10,458.08(19,814.17)$ & $3,944.3(2,112.25-7,933.50)$ & $7,673.38(17,576.56)$ & $2,768.86(0-6,229.93)$ \\
\hline Reduced productivity & $176(61.3)$ & $60.6(78.0)$ & $12,462.87(15,411.53)$ & $8,023.28(3,360.71-13,844.28)$ & $9,766.22(14,570.92)$ & $5,537.71(1,290-11,764.80)$ \\
\hline Unemployment due to migraine & $45(15.7)$ & $220.3(78.6)$ & $16,525.20(19,367.00)$ & $9,166.69(4,496.57-28,553.83)$ & $347.90(3,407.57)$ & $0(0-0)$ \\
\hline Short-term disability & $39(13.6)$ & $61.9(66.6)$ & $7,695.10(5,858.46)$ & $7,234.32(2,773.50-12,113.75)$ & $1,019.83(3,349.19)$ & $0(0-0)$ \\
\hline Long-term disability & $47(16.4)$ & $213.8(72.3)$ & $32,679.34(20,098.83)$ & $36,675.99(12,900-44,993.91)$ & $1,943.10(9,055.53)$ & $0(0-0)$ \\
\hline
\end{tabular}

$\mathrm{CAD}=$ Canadian dollar; $\mathrm{IQR}=$ interquartile range; $\mathrm{n}=$ number; $\mathrm{SD}=$ standard deviation.

${ }^{1}$ Across entire study sample.

Data source: patient survey.

an updated and comprehensive assessment of the economic burden of illness associated with migraine in Canada as well as impact of this disease on QoL.

Our findings demonstrate that migraine-related disability and health outcomes result in a substantial economic burden to both patients and the healthcare system, directly from healthcare and nonhealthcare costs, such as prescription medications and transportation to medical appointments, and indirectly through loss of work productivity. The total estimated annual cost of $\mathrm{CM}$ is $\$ 25,669$ per patient, while the annual total costs for high-frequency EM (8 to 14 MMDs) and low-frequency EM (4 to 7 MMDs) migraine were estimated to be $\$ 24,885$ and $\$ 15,651$, respectively. Interestingly, our results showed an existing intra-variability of the burden of migraine within the Canadian EM patient population, where high-frequency EM patients undergo a significant burden that is as high as that observed for the CM patients. Interestingly, this observation is consistent with other recent studies showing that patients with 8 to 14 MMDs are as disabled as patients with ICHD-3 defined CM, highlighting the need to take into account these observations in upcoming revisions of the diagnostic criteria for $\mathrm{CM}^{6}$ While it is still important, the economic burden observed for low-frequency EM patients is considerably lower than that for high-frequency episodic and $\mathrm{CM}$ patients.

Prescription medications and HCP visits account for the majority of direct healthcare costs. Given that treatment of migraine is aimed to reduce the burden of disease, rather than being curative, the necessity 
Table 9: Summary of total costs associated with migraine by migraine type ( $n=274$; low-frequency EM was defined as 4-7 MMDs, high-frequency EM was defined as 8-14 MMDs, and chronic migraine was defined as 15 + MMDs)

\begin{tabular}{|c|c|c|c|c|c|c|c|c|c|}
\hline \multirow[b]{3}{*}{ Treatment } & \multicolumn{9}{|c|}{ Overall annual cost per migraine patient ${ }^{1}$} \\
\hline & \multicolumn{3}{|c|}{ Low-frequency EM $(n=70)$} & \multicolumn{3}{|c|}{ High-frequency EM $(n=101)$} & \multicolumn{3}{|c|}{ Chronic migraine $(n=103)$} \\
\hline & $\begin{array}{c}\text { Any } \\
\text { utilization, } n \\
(\%)\end{array}$ & Mean (SD) & Median $(\mathbf{I Q R})^{2}$ & $\begin{array}{c}\text { Any utilization, } n \\
(\%)\end{array}$ & Mean (SD) & Median $(\mathbf{I Q R})^{2}$ & $\begin{array}{c}\text { Any utilization, } n \\
(\%)\end{array}$ & Mean (SD) & Median (IQR) ${ }^{2}$ \\
\hline
\end{tabular}

Direct costs

Direct healthcare costs

\begin{tabular}{|c|c|c|c|c|c|c|c|c|c|}
\hline $\begin{array}{l}\text { Prescription } \\
\text { medications }\end{array}$ & $70(100.0)$ & $4,509.89(3,607.20)$ & $\begin{array}{c}3,736.80(1,011.60- \\
7,291.80)\end{array}$ & $100(99.0)$ & $6,423.47(4,051.15)$ & $\begin{array}{c}6,607.20(3,138.00- \\
9,624.96)\end{array}$ & $103(100.0)$ & $8,588.55(5,559.72)$ & $\begin{array}{c}8,616.00(3,564.00- \\
12,459.36)\end{array}$ \\
\hline $\begin{array}{l}\text { Nonprescription } \\
\text { treatments }\end{array}$ & $33(47.1)$ & $7.91(28.35)$ & $0(0-2.88)$ & $44(43.6)$ & $14.18(43.02)$ & $0(0-0)$ & $34(33.0)$ & $16.22(63.83)$ & $0(0-0)$ \\
\hline $\begin{array}{l}\text { Healthcare } \\
\text { providers }^{3}\end{array}$ & $68(97.1)$ & $2,043.09(1,722.22)$ & $\begin{array}{c}1,577.36(864.88- \\
2,800.84)\end{array}$ & $101(100.0)$ & $2,099.33(2,255.68)$ & $\begin{array}{c}1,320.36(728.16- \\
2,621.96)\end{array}$ & $103(100.0)$ & $3,283.49(4,342.80)$ & $\begin{array}{c}1,866.48(910.20- \\
4,021.44)\end{array}$ \\
\hline Laboratory tests & $19(27.1)$ & $9.29(16.12)$ & $0(0-30.97)$ & $35(34.7)$ & $17.17(36.24)$ & $0(0-30.97)$ & $34(33.0)$ & $21.95(50.13)$ & $0(0-30.97)$ \\
\hline $\begin{array}{l}\text { Nonsurgical } \\
\text { procedures/ } \\
\text { interventions }^{4}\end{array}$ & $42(60.0)$ & $684.53(456.56)$ & $\begin{array}{c}635.55(381.33- \\
1,076.79)\end{array}$ & $62(61.4)$ & $550.70(441.38)$ & 499.48 (181.70-772.77) & $68(66.0)$ & $602.69(535.42)$ & $\overline{484.34(127.11-783.12)}$ \\
\hline Devices & $3(4.3)$ & 23.26 (194.58) & $0(0-0)$ & $7(6.9)$ & $46.69(247.93)$ & $0(0-0)$ & $9(8.7)$ & $105.22(376.99)$ & $0(0-0)$ \\
\hline $\begin{array}{l}\text { Total direct } \\
\text { healthcare costs }{ }^{1}\end{array}$ & & \multicolumn{2}{|c|}{$7,004.16$} & & \multicolumn{2}{|c|}{$8,938.89$} & & \multicolumn{2}{|c|}{$12,413.31$} \\
\hline \multicolumn{10}{|c|}{ Direct non-healthcare costs } \\
\hline $\begin{array}{l}\text { Childcare provider/ } \\
\text { paid help }\end{array}$ & $7(10.0)$ & $3,475.20(2,711.54)$ & $\begin{array}{c}3,366.40(1,190.40- \\
5,760.00)\end{array}$ & $4(4.0)$ & $17,152.00(11,457.97)$ & $\begin{array}{c}11,520.00(9,600.00- \\
30,336.00)\end{array}$ & $8(7.8)$ & $6,194.67(4,366.80)$ & $\begin{array}{c}7,184.00(1,280.00- \\
9,360.00)\end{array}$ \\
\hline $\begin{array}{l}\text { Transportation for } \\
\text { medical } \\
\text { appointments }\end{array}$ & $59(84.3)$ & $469.66(1,214.98)$ & $0(0-200)$ & $87(86.1)$ & $1,507.78(7,292.54)$ & $0(0-233.84)$ & $92(89.3)$ & $300.42(1,499.35)$ & $0(0-71.55)$ \\
\hline $\begin{array}{l}\text { Total direct non- } \\
\text { healthcare costs }{ }^{1}\end{array}$ & & \multicolumn{2}{|c|}{576.82} & & \multicolumn{2}{|c|}{$2,017.24$} & & \multicolumn{2}{|c|}{585.16} \\
\hline \multicolumn{10}{|l|}{ Indirect costs } \\
\hline Lost productivity & $56(80.0)$ & $10,658.97(16,792.45)$ & $\begin{array}{c}6,321.00(1,256.98- \\
14,241.60)\end{array}$ & $87(86.1)$ & $16,170.05(32,757.17)$ & $6,594.56(0-15,443.12)$ & $81(78.6)$ & $16,313.17(31,915.13)$ & $0(0-20,863.06)$ \\
\hline Total indirect costs & & \multicolumn{2}{|c|}{$\begin{array}{l}8,070.37 \\
\end{array}$} & & \multicolumn{2}{|c|}{$13,928.66$} & & \multicolumn{2}{|c|}{$12,670.42$} \\
\hline
\end{tabular}




\begin{tabular}{|c|c|c|c|c|c|c|c|c|c|}
\hline \multirow[b]{3}{*}{ Treatment } & \multicolumn{9}{|c|}{ Overall annual cost per migraine patient ${ }^{1}$} \\
\hline & \multicolumn{3}{|c|}{ Low-frequency EM $(n=70)$} & \multicolumn{3}{|c|}{ High-frequency EM $(n=101)$} & \multicolumn{3}{|c|}{ Chronic migraine $(n=103)$} \\
\hline & $\begin{array}{c}\text { Any } \\
\text { utilization, } n \\
(\%)\end{array}$ & Mean (SD) & Median (IQR) ${ }^{2}$ & $\begin{array}{c}\text { Any utilization, } n \\
(\%)\end{array}$ & Mean (SD) & Median $(\mathrm{IQR})^{2}$ & $\begin{array}{c}\text { Any utilization, } n \\
(\%)\end{array}$ & Mean (SD) & Median (IQR) ${ }^{2}$ \\
\hline $\begin{array}{c}\text { Total direct and } \\
\text { indirect costs }\end{array}$ & & \multicolumn{2}{|c|}{$\frac{1}{15,651.34}$} & & \multicolumn{2}{|c|}{$\frac{1}{24,884.79}$} & & \multicolumn{2}{|c|}{$25,668.89$} \\
\hline
\end{tabular}

EM, episodic migraine; IQR = interquartile range; $\mathrm{n}=$ number; $\mathrm{SD}=$ standard deviation.

${ }^{1}$ Across entire study sample.

${ }^{2}$ For patients with no utilization and $\$ 0$ in costs, the Q1 in IQR is 0 .

${ }^{3}$ Healthcare providers direct cost considers frequency and costs of healthcare provider visits for migraine patients in Canada, over the 12 months prior to study enrollment (e.g. neurologist, nurse practitioner, ophthalmologist, massage therapist, and acupuncturist).

${ }^{4}$ Nonsurgical procedures considers frequency and costs of nonsurgical interventions for migraine patients in Canada, over the 12 months prior to study enrollment (e.g.. MRI, CT scan, spinal tap, and botox injection procedure).

*Low-frequency EM is defined as an average of 4-7 migraine days per month; high-frequency EM is defined as an average of $8-\leq 14$ migraine days per month; chronic migraine is defined as an average of $\geq 15$ migraine days per month.

Low-frequency EM: summary of total costs were calculated for those patients who reported the required information including hours of work and wages. About $58 \%$ of patients provided information to calculate total direct healthcare costs, while $38 \%$ and $91 \%$ of patients contributed toward calculating total direct non-healthcare costs and total indirect costs, respectively.

High-frequency EM: summary of total costs were calculated for those patients who reported the required information including hours of work and wages. About 53\% of patients provided information to calculate total direct healthcare costs, while $21 \%$ and $97 \%$ of patients contributed toward calculating total direct non-healthcare costs and total indirect costs, respectively.

CM: summary of total costs were calculated for those patients who reported the required information including hours of work and wages. About $54 \%$ of patients provided information to calculate total direct healthcare costs, while $3 \%$ and $77 \%$ of patients contributed toward calculating total direct non-healthcare costs and total indirect costs, respectively. 
for prolonged utilization was a key driver of direct annual healthcare costs. Interestingly, our results show that the direct annual healthcare cost for both chronic and EM patients is significantly higher than reported previously. ${ }^{11}$ However, this could be explained by numerous differences in study design. Our study shows that the costs of some healthcare services that were not assessed in previous studies, such as physiotherapy, massage therapy, and naturopathy, had a total annual cost of $\$ 4,610$ and could significantly impact the total direct healthcare costs. In addition, our study was able to access medical records and looked back to the previous 12 months, while the Stokes paper considered 3-month patients' recall to prevent biases. ${ }^{11}$

Direct non-healthcare costs, such as childcare and transportation to medical appointments, added to the economic burden of migraine for patients suffering from both episodic and CM although not all patients reported childcare-related costs. It may be likely that family support or other supports not captured in our study are utilized when needed to help with childcare during migraine attacks.

Using a comprehensive patient survey, our results showed that indirect costs resulting from loss of work productivity have a major economic impact on the burden of migraine for both episodic and $\mathrm{CM}$ patients. Indirect costs accounted for more than $50 \%$ of the total economic burden reported for both episodic and CM patients. These results will help with our understanding and awareness of the migraine burden, since indirect costs due to loss of work productivity have not been assessed in previous studies.

Patients in Canada who suffer from CM also typically suffer from depression and anxiety. Based on validated PRO instruments, we observed that $75.6 \%$ of respondents reported some level of anxiety and depression, among which $38 \%$ was moderate to severe intensity. Both the validated PRO instruments and the patient survey showed that migraine patients experience important disability in their daily lives due to migraine. Furthermore, an overall reduction of nearly half, in terms of work productivity, was experienced due to migraine.

Although there is limited Canadian data available, our findings are aligned with recent findings reported by Stokes et al. ${ }^{11}$ They found that CM caused greater headache-related disability, greater impairment of QoL, and increased resource utilization compared to EM. However, our results showed that patients with high-frequency EM (8 to 14 MMDs) can impose an overall burden that is as important as that for CM.

\section{Limitations}

The differentiation between tension headaches and migraines can be challenging when classifying headaches from headache diary entries alone and was considered during study protocol development. When a headache was recorded as moderate to severe, it was counted as a migraine day since tension headaches in these patients tend to be relatively mild or described as 'background headaches'. We also considered the requirements for CM, which by ICHD-III defines as at least 15 headache days per month, with at least 8 migraine days. Differentiating between migraine and tension headache was difficult if there was no diary information available and no mention of exact frequencies in the medical chart. This was a limitation of the data collected.
Although the burden of both EM and CM was assessed in this study, we acknowledge that enrolling patients from five headache-specialized centers could introduce a bias on the assessed overall burden of migraine, as the migraine patient population from these centers may be representative of the most severe and disabled EM and CM patients. Therefore, the results may not be generalizable to the overall migraine population but would be applicable for migraine patients seen at headache clinics across Canada.

Another limitation unique to this study was related to the limited data available on erenumab use. The study coincided with the launch of erenumab in Canada, which is a new class of treatment for migraine. There is a limited amount of available data on erenumab retrospectively, and therefore the overall assessment for the burden of migraine could potentially be biased with the inclusion of erenumab in the present study. Furthermore, the exclusion of erenumab could also bias the results, given that $54.4 \%$ of patients in the study population had been treated with erenumab at some point, and that it is our assumption that patients on erenumab were more severe patients. Additional follow-up data on erenumab and other treatments in the calcitonin gene-related peptide (CGRP) inhibitor class are necessary to better assess the burden of illness associated with migraine in Canada. Typically, the benefits of this class are observed after 3 to 6 months of use. Therefore, it is likely that benefits with respect to resource use would also see an equivalent lag period, further underscoring the need for data on long-term use of this new class in routine clinical practice.

Additional limitations common to these types of studies include variations in unit costs as they come from publicly available sources and no sensitivity analyses were conducted on key cost input parameters. It was also necessary to make several assumptions when interpreting and analyzing the data, which would also contribute to variability in results. Nevertheless, this study provides an updated and comprehensive assessment of the burden of illness related to migraine in Canada. This study also provides robust estimates for direct and indirect costs associated with migraine through its mixed-methods approach.

\section{Conclusions}

This study provides a recent and comprehensive assessment of the burden of illness associated with migraine in Canada. Clinical, economic, and humanistic burden were assessed through a retrospective chart review and cross-sectional patient survey. We observed that migraine was associated with moderate to severe disability and often accompanied by depression and anxiety. This results in substantial economic burden, directly from healthcare costs such as prescription medications and indirectly through lost work productivity. We also observed that high-frequency EM patients experience significant burden, similar to that observed for CM patients. Future studies will be needed to assess the impact of the emerging CGRP-based migraine preventive therapies on the burden of migraine in Canada.

\section{ACKNOWLEDGMents}

The authors would like to thank Robert Sambrook, Rajvi Wani, Julie Blouin, Christian Kindundu, and Natacha Bastien for their support. 


\section{Funding}

This study was funded by Novartis Canada.

\section{Disclosures}

ZK and MO are employees of ICON plc, a company contracted by Novartis for this study.

DR and SS are employees of Novartis, may hold stock or options.

FA has received speaker's honoraria from TEVA, Eli Lilly, Novartis, Allergan, Aralez, and ICEBM, consultancy fees from TEVA, Eli Lilly, Novartis, Lundbeck, and ICEBM, and sponsorship for research/clinical trials from Eli Lilly, Allergan, Novartis, and TEVA.

\section{Statement of Authorship}

All authors contributed to the study design and development of the study protocol. ZK and MO were responsible for the conduct of all data collection and analysis activities. All authors contributed to the development and review of the manuscript. Specifically, ZK and MO developed the manuscript draft with close collaboration with DR and SS. FA provided scientific and clinical input throughout the design and conduct of the study as well as during the writing of the manuscript.

\section{SuPPlementary Material}

To view supplementary material for this article, please visit https://doi.org/10.1017/cjn.2021.75.

\section{REFERENCES}

1. Vo P, Khurana, V. Literature review: epidemiology, disease progression, burden of disease, treatment patterns, unmet needs and real world evidence gaps in migraine. [Internal document]. In press 2017.

2. Lipton RB. Chronic migraine, classification, differential diagnosis, and epidemiology. Headache. 2011;51:77-83.

3. Stovner L. Global, regional, and national burden of migraine and tension-type headache, 1990-2016: a systematic analysis for the Global Burden of Disease Study 2016. Lancet Neurol. 2018;17:954-76.

4. Ramage-Moring PL, Gilmour H. Prevalence of migraine in the Canadian household population. Statistics Can. Health Reports. 2014;25:10-16.

5. IHS. The international classification of headache disorders, 3rd edition. (beta version). Cephalalgia. 2013;33:629-808.

6. Chalmer MA, Hansen TF, Lebedeva ER, Dodick DW, Lipton RB, Olesen J. Proposed new diagnostic criteria for chronic migraine. Cephalalgia. 2020;40:399-406.

7. Lipton RB, Pavlovic JM, Haut SR, Grosberg BM, Buse DC. Methodological issues in studying trigger factors and premonitory features of migraine. Headache. 2014;54:1661-69.

8. Cooke LJ, Becker WJ. Migraine prevalence, treatment and impact: the Canadian women and migraine study. Can J Neurol Sci. 2010;37:580-87.

9. Krayl M, Pegg E., Kulyk I., O’Brien E., Snellman J. Non-interventional study protocol CAMG334A3301: The Burden of migraine in specialist headache centers treating patients with prophylactic treatment failure. [Internal Document]. In press 2017.

10. Migraine Canada. Disability and Migraine 2020 [cited 2020]. Available from: https://migrainecanada.org/disability-andmigraine/

11. Stokes M, Becker WJ, Lipton RB, et al. Cost of health care among patients with chronic and episodic migraine in Canada and the USA: results from the International Burden of Migraine Study (IBMS). Headache. 2011;51:1058-77. 